\title{
$\begin{array}{ll}\text { Research Square } & \text { Preprints are preliminary reports that have not undergone peer review. }\end{array}$ or referenced by the media as validated information. \\ New point mutation in CREBBP Gene cause Rubinstein-Taybi syndrome: A case report
}

\section{Dan Wang}

Hunan Children's Hospital https://orcid.org/0000-0002-0292-3138

$\mathrm{Na} \mathrm{Liu}$

Second Xiangya Hospital

Yunbin Xiao ( $\nabla$ xiaoyunbinrui@126.com )

Second Xiangya Hospital

Zhenyu Liao

Hunan Children's Hospital

Zhenghui Xiao

Hunan Children's Hospital

Zhi Chen

Hunan Children's Hospital

Xun Wang

Hunan Children's Hospital

\section{Case report}

Keywords: CREBBP, Rubinstein-Taybi syndrome, case report

Posted Date: November 19th, 2020

DOl: https://doi.org/10.21203/rs.3.rs-110594/v1

License: (c) (i) This work is licensed under a Creative Commons Attribution 4.0 International License.

Read Full License 


\title{
New point mutation in CREBBP Gene cause Rubinstein-Taybi syndrome: A case report
}

Dan Wang ${ }^{1 *}$, Na Liu ${ }^{2 *}$, Yunbin Xiao ${ }^{2}$, Zhenyu Liao $^{3}$, Zhenghui Xiao ${ }^{4}$, Zhi Chen ${ }^{1}$, Xun Wang ${ }^{1}$

\# Wang Dan and Liu Na contributed equally to this work

*Corresponding Author

Yunbin Xiao

Department of Cardiology

Hunan Children's Hospital

No. 86 Ziyuan Road, Yuhua District,

Changsha, Hunan, 410007, PR. China

Tel: +86-0731-85356800

Fax: +86-0731-85350908

E-mail: xiaoyunbinrui@126.com

\begin{abstract}
Background: RSTS is a rare autosomal dominant inheritance disease. It is easy to overlap with the phenotypes of other syndromes, To assist with future diagnoses, we summarize the clinical and genetic characteristics of children with Rubinstein-Taybi syndrome.

Case presentation: The patient, female, aged 3 months, $4.2 \mathrm{~kg}$, was admitted into our hospital 3 times after birth due to repeated infections, shortness of breath, poor response, low crying, cyanosis and poor breastfeeding. The child has a special complexion with congenital heart disease, hearing impairment, and hypothyroidism. The anterior fontanel has a lot of vellus hair, mainly on the back, low hairline, micrognathia, high palate arch. the high-precision clinical explicit PLUS test and analysis were performed on all of their blood. CREBBP gene heterozygous mutation c.890T> A (p.L297*) was detected. At the same time, the sequencing data showed that the parents of the examinee did not carry this mutation, which may be new.
\end{abstract}

Conclusion: combining clinical manifestations and genetic testing can clearly diagnose Rubinstein-Taybi syndrome and enriched human CREBBP gene mutation database.

Keywords: CREBBP, Rubinstein-Taybi syndrome, case report 


\section{Background}

Rubinstein-Taybi syndrome (RSTS) was first reported by Rubinstein and Taybi in $1963^{[1]}$. RSTS is a rare autosomal dominant inheritance disease with an estimated prevalence of 1:125.000 in live births ${ }^{[2]}$. The main clinical presentations are congenital multi-system abnormalities such as growth retardation, intellectual disability, microcephaly, broad thumbs and halluces, distinctive facial features, tumor ${ }^{[3-4]}$. Currently, The diagnosis rate and clinical presentations of RSTS are rare. It is easy to overlap with the phenotypes of other syndromes so as to make clinical diagnosis challenging. RSTS can be divided into two categories (including CREBBP and EP300) according to different pathogenic genes, Among them, CREBBP mutation is the main pathogenic gene (accounting for $55 \%-60 \%$ of cases), while EP300 gene mutation accounts for $8 \%$ of the causes of RSTS patients ${ }^{[5]}$. Until now, a variety of CREBBP-induced lesions have been reported, including point mutations (30\%-50\%) and large deletions (10\%). Although there is some understanding of clinical, genetic and allelic heterogeneity, a clear genotype-phenotype correlation has not been established. This paper mainly reports on RSTS in infant caused by new point mutations in the CREBBP gene in order to broaden the molecular knowledge about the major RSTS gene, as well as to find more clinical manifestations.

\section{Case presentation}

The patient, female, aged 3 months, $4.2 \mathrm{~kg}$, was admitted into our hospital 3 times after birth due to repeated infections, shortness of breath, poor response, low crying, cyanosis and poor breastfeeding. The patient has developmental retardation. The child has a special complexion with congenital heart disease, hearing impairment, and hypothyroidism. The patient, G1P1 and 35+2 weeks pregnant, was born in a local maternity and child health center. The birth weight was $2.18 \mathrm{Kg}$ with clear amniotic fluid and normal placenta. There was premature rupture of the membrane 14+ hours, and cyanosis when she was born, Apgar score is unknown. Her mom had a cold during $1+$ month of pregnancy. She took some medicine, but she forgot it . The symptom was improved after 7 days. In the first trimester of pregnancy, Hyperthyroidism was untreated by reexamination of normal nail function after 3 weeks. Diabetes at 28 weeks of pregnancy was shown, but it is in a normal level by diet control, good blood sugar control. There were gastroenteritis at 32 weeks of gestation, and local hospitals provide fetal protection. The specific 
medication is unknown. There is no exposure to poisons, and there is no pregnancy hypertension during pregnancy.

Physical examination: clear mind, unresponsiveness, and low crying. The anterior fontanel is soft, $1.5^{*} 1.5 \mathrm{~cm}$ in size, with a lot of vellus hair, mainly on the back, low hairline, micrognathia, small mandible, high palate arch, cyanosis around the lips, obvious throat sounds. The heart rate was 140 beats $/ \mathrm{min}$. The abdomen is soft. The liver is $1 \mathrm{~cm}$ below the right rib of the liver. The spleen is below the costal rib. Hypotonia, broad thumbs and toes. (Figure 1, Figure2).

Auxiliary examination: Cardiac Color Doppler ultrasound: 1. Atrial shunt: (6mm) 2. The ascending aorta widens; the right atrium and right ventricle are large. 3. Patent ductus arteriosus. 4. Aortic valve acoustic image changes: suspected second-valve deformity? 5. Normal left heart function. Cranial MRI: 1. Hemosiderin in the cerebellar curtain, straight sinus, and posterior longitudinal fissure zone; 2. Brain changes in premature infants; 3. Forehead: The display is slightly narrow, the bilateral ventricular body is slightly full, and the bilateral basal ganglia area shows the contralateral slightly shorter T1 signal shadow.

Hearing impairment: short sound stimulates the left and right ears with $120 \mathrm{dBspl}$ respectively, the waveform differentiation on the right side is prolonged, the latency of I, III and V waves is prolonged, the interval of I-III, IV and III-V waves is prolonged, and the threshold of V-wave response is $75 \mathrm{spldb}$. The left ABR is basically normal, and the V-wave response threshold is 65 spldb. The right auditory conduction pathway is involved.

Chest radiograph: enlarged heart shadow, pneumonia.

Fundus examination: The omentum below the right eye shows a rounded pigment disorder of myopia, the left eye papillary traits are irregular, the blood vessels can run, the black nipple changes can be seen, and the visible area is flat.

Abnormal hematuria screening was negative. A axis five items: thyroid stimulating hormone $0.073 \mathrm{uIU} / \mathrm{ml}$, triiodothyronine $1.33 \mathrm{nmol} / \mathrm{L}$, thyroxine $144.8 \mathrm{nmol} / \mathrm{L}$, free T3 $3.31 \mathrm{pmol} / \mathrm{L}$, Free thyroxine $20.32 \mathrm{pmol} / \mathrm{L}$. NT-ProBNP13031pg/ml. Repeated positive sputum culture suggests Escherichia coli infection. Full set of immunizations suggest hypocomplementemia. Lymphocyte subsets suggest a decrease in the number of total $\mathrm{T}$ lymphocytes, and suppressive/cytotoxic $\mathrm{T}$ lymphocytes. Head color Doppler ultrasound showed enhanced brain parenchymal echo (PFE 1 degree) and right choroid plexus cyst. ECG prompts sinus tachycardia. Total bilirubin 
187.70umol/L. Fiberoptic bronchoscopy suggests laryngeal cartilage dysplasia and tracheal softening. It is recommended to improve the lung CT scan in order to make sure whether there is local compression.

According to the examination results, genetic metabolic diseases are considered, and related genetic examinations are sent.

With the informed consent of their parents, $2 \mathrm{ml}$ of blood samples was collected from the patient and her parents using vacuum-EDTA tubes. Genomic DNA was extracted from peripheral blood using the Solpure Blood DNA kit (Magen)according to the manufacturer's instructions. The genomic DNA of the three patients was then fragmented by Q800R Sonicator (Qsonica) to generate $300-500 \mathrm{bp}$ insert fragments.

In this genetic test, we used the high accuracy clinical manifestation PLUS test and analysis of the test recipient, and his father and mother were performed simultaneously. Sequence variants were annotated using population and literature databases including 1000 Genomes, dbSNP, GnomAD, Clinvar, HGMD and OMIM. And CREBBP was detected. Gene heterozygous variation c.890T> A (p.L297*)(figure 3). It means that the nucleotide 890 is changed from thymine nucleotide to adenine nucleotide, and the mutation is a splice site mutation. At the same time, the sequencing data showed that the parents of the examinee did not carry this mutation, and this mutation may be new. Meanwhile, through copy number and SNP analysis, no copy number variation that may be related to clinical manifestations was detected. It is the first report that no related records have been found in the Thousand People Database, dbSNP Database and HapMap Database.

Within 3 months after birth, she was hospitalized repeatedly 5 times, including 3 times in our hospital. During hospitalization, he was repeatedly given nasal catheter oxygen or CAPA non-invasive auxiliary ventilation, phototherapy, expectorant, bronchoscopic lavage, trophic nerve, diuretic diuretic, nebulization, repeated anti-infection and other treatments. Now she is 8 months, the head is still not stable. She can't make a sound of "ah" and take the initiative to grab things, and the laugh is not obvious.

\section{Discussion}

RSTS is a rare congenital disease with an autosomal dominant disorder. $90 \%$ of cases are new mutations. Most cases are sporadic, and family clustering cases are rare. Most of the children can 
survive to adulthood. There are currently more than 1,000 cases reported abroad, but few domestic report. Yu S reported 18 RSTS patients including one splicing variants, two missense, four nonsense, five frameshift, and seven intragenic deletions, Others are mainly case reports ${ }^{[6]}$. This report aimed to study new point variant of CREBBP and broaden the genotype-phenotype correlation.

Approximately $60 \%$ of RSTS is caused by CREBBP(16p13, OMIM\#600140) gene mutation, which is RSTS-1; $10 \%$ is caused by EP300 gene mutation, which is RSTS- $2^{[7]}, 30 \%$ of which have not yet found a disease-causing gene. The clinical manifestations are mainly facial dysmorphic features like prominent forehead, low anterior hairline and grimacing smile, microcephaly, short stature, high arched eyebrows, long eyelashes, oblique eyelids, wide nose bones, high palate and minor jaw deformity. With broad thumbs and/or halluces. mild to severe intellectual disability. It can also be accompanied by cataract, congenital heart disease, abnormal kidney function, cryptorchidism and tumor. The pathogenic mutations of this gene-related disease are mainly new mutations (PMID: 20301699, 16868563) ${ }^{[8]}$.

In this report, the patient's main performance is low anterior hairline, small jaw, high palatal arch, broad thumbs and halluces, congenital heart disease, Intellectual disability, growth retardation and dyskinesia, mainly hirsutism, Hypoacusia ${ }^{[9]}$, endocrine anomalies like hypothyroidism ${ }^{[10]}$, which is consistent with previous reports. But fundus problems have not been reported before.

The CREBBP gene is located on chromosome 16p13.3, with a total length of $156 \mathrm{~kb}$, and a total of 31 exons (transcript: NM_0043380.2). EP300 (22q13, OMIM \#402700) is a homologous gene of CREBBP gene, located on chromosome 22q13.2. The total length is about $88 \mathrm{~kb}$, with 31 exons (transcript: NM_001429.3). The CREBBP gene encodes the CREB-binding protein (CBP), and the EP300 gene encodes E1A-associated protein p300. P300/CBP are all histone acetyltransferases, interacting with more than 400 proteins, and play an important role in embryonic development and cell growth and differentiation ${ }^{[11]}$. P300 / CBP mainly plays a role by regulating gene expression and multiples signaling pathways in the cell, and participates in DNA replication and repairs through acetylation of more than 70 non-histone proteins ${ }^{[12]}$; it also forms a complex with MDM2 protein, through polyubiquitin ligase activity Activate P53.

CREBBP deletion ranges from one exon to the entire gene. At least $50 \%$ patients take along 
the deletion of five or more exons. One exon of this patient is changing. The new heterozygous variation of CREBBP gene c.890T> A (p.L297*) has not been reported in relevant clinical cases. The region where the mutation is located is an important part of this protein. The amino acid sequence of different species is highly conservative, and the prediction may cause that the termination code for amino acids appears in advance. Therefore, the CBP protein is truncated and the glutamic acid-rich region is deleted, which affect the function of the CBP protein. So far, this variation has not been reported in our reference population gene database. To sum up, according to the clinical manifestations of the examinee and the analysis of the family, and based on the US ACMG Variant Classification Guidelines (PMID: 25741868), this mutation is "category 1-pathogenic mutation".

At present, the treatment for RSTS patients is only symptomatic treatment, Including physiotherapy, ergonomic therapy, tube feeding, antibiotics to prevent airway infections and surgical treatment for heart or kidney deformities ${ }^{[13]}$. Besides ,they usually have a social adaptation disorder which need early intervention ${ }^{[14]}$. So the sooner the diagnosis is , the better. As for this child, the diagnosis was early and some symptoms may don't show up now. And the most important thing is to prevent the birth of this kind of children.

\section{Conclusion}

Despite previous research reports that the size and the severity of the disease is related, and some children die shortly after birth ${ }^{[15]}$. But the protein structure changes caused by different mutation points are different, and the resulting clinical manifestations are also different, the genotype-phenotype correspondence relationship is currently inconclusive ${ }^{[16]}$. Children with RSTS need a multi-system evaluation after clinical diagnosis. For example, the variant c.3837-2A $>\mathrm{C}$ can cause fetal phenotype ${ }^{[17]}$. In conclusion, combining clinical manifestations and genetic testing can clearly diagnose Rubinstein-Taybi syndrome and enriched human CREBBP gene mutation database.

\section{Abbreviations}

RSTS: Rubinstein-Taybi syndrome. CBP: CREB-binding protein.

\section{Acknowledgements}


No acknowledgements

\section{Funding}

This work supported by hospital-level research funding(No.201939c), Key Laboratory Platform Project (No.2018TP1028) and 2019 Hunan Province Traditional Chinese Medicine Research Program (Project Item number 201914)

\section{Author contributions}

Conceptualization: Yunbin Xiao, Zhenyu Liao, Zhenghui Xiao, Xun Wang. Data curation: Yunbin Xiao. Formal analysis: Yunbin Xiao. Investigation: Zhi Chen. Methodology: Xun Wang. Resources: Zhenyu Liao. Supervision: Zhi Chen, Zhenghui Xiao. Validation: Yunbin Xiao. Visualization: Zhenghui Xiao. Writing - Original Draft: Dan Wang. Writing - Review \& Editing: $\mathrm{Na}$ Liu

\section{Consent for publication}

This study was approved by the institutional review boards of Hunan Children's Hospital where the patient data were collected. Written informed consent was obtained from the patient's parents for publication of this case report and accompanying images.

\section{Competing interests}

The authors declare that they have no competing interests.

\section{Publisher's Note}

Springer Nature remains neutral with regard to jurisdictional claims in published maps and institutional affiliations.

\section{Author details}

1 Department of Cardiology, Hunan Children's Hospital, Changsha, Hunan Province, 410007,China

2 Department of Cardoilogy, the Second Xiangya Hospital, Central South University, Changsha 410011, China

3 Neonatology department, Hunan Children's Hospital, Changsha, Hunan Province, 410007, China 4 Intensive Care Unit, Hunan Children's Hospital, Changsha, Hunan Province, 410007, China

\section{References}


[1] Rubinstein JH, Taybi H. Broad thumbs and toes and facial abnormalities. A possible mental retardation syndrome. Am J Dis Child. 1963;105:588-608.

[2] Korzus E. Rubinstein-Taybi Syndrome and Epigenetic Alterations. Adv Exp Med Biol. 2017;978:39-62.

[3] Boot MV, van Belzen MJ, Overbeek LI, Hijmering N, Mendeville M, Waisfisz Q, Wesseling P, Hennekam RC, de Jong D. Benign and malignant tumors in Rubinstein-Taybi syndrome. Am. J. Med. Genet. A. 2018;176, 597-608.

[4] Pérez-Grijalba V, García-Oguiza A, López M, et al. New insights into genetic variant spectrum and genotype-phenotype correlations of Rubinstein-Taybi syndrome in 39 CREBBP-positive patients. Mol Genet Genomic Med. 2019;7:e972.

[5] Spena S, Gervasini C. Ultra-Rare Syndromes: The Example of Rubinstein-Taybi Syndrome. J Pediatr Genet. 2015;4:177-186.

[6] Yu S, Wu B, Qian Y, et al. Clinical exome sequencing identifies novel CREBBP variants in 18 Chinese Rubinstein-Taybi Syndrome kids with high frequency of polydactyly. Mol Genet Genomic Med. 2019;7(12):e1009.

[7] Lopez M, Garcia-Oguiza A, Armstrong J, et al. Rubinstein-Taybi 2 associated to novel EP300 mutations: deepening the clinical and genetic spectrum. BMC Med Genet. 2018;19(1):36. [8] Spena S, Gervasini C. Ultra-Rare Syndromes: The Example of Rubinstein-Taybi Syndrome. J Pediatr Genet. 2015;4:177-186.

[9] Wincent J, Luthman A, van Belzen M, et al. CREBBP and EP300 mutational spectrum and clinical presentations in a cohort of Swedish patients with Rubinstein-Taybi syndrome. Mol Genet Genomic Med. 2016;4(1):39-45.

[10] Kurtoglu S, Akcakus M, Gunes T, et al. Congenital hypothyroidism associated with Rubinstein-Taybi syndrome.

J Pediatr Endocrinol Metab. 2003;16(3):457-459.

[11] Korzus E. Rubinstein-Taybi Syndrome and Epigenetic Alterations.Adv Exp Med Biol. 2017;978:39-62.

[12] Dutto I,Scalera C,Tillhon M,et al.Mutations in CREBBP and EP300 genes affect DNA repair of oxidative damage in Rubinstein-Taybi syndrome cells. Carcinogenesis. 2020;41(3):257-266. 
[13] Papathemeli D, Schulzendorff N, Kohlhase J, et al. Pilomatricomas in Rubinstein-Taybi syndrome. J Dtsch Dermatol Ges. 2015;13(3):240-242.

[14] Crawford H, Moss J, Groves L, et al. A Behavioural Assessment of Social Anxiety and Social Motivation in Fragile X, Cornelia de Lange and Rubinstein-Taybi Syndromes. J Autism Dev Disord. 2020;50(1):127-144.

[15] Rusconi D, Negri G, Colapietro P, et al. Characterization of 14 novel deletions underlying Rubinstein-Taybi syndrome: an update of the CREBBP deletion repertoire. Hum Genet. 2015, 134(6): 613-626.

[16] Negri G, Magini P, Milani D, et al. Exploring by whole exome sequencing patients with initial diagnosis of Rubinstein-Taybi syndrome: the interconnections of epigenetic machinery disorders. Hum Genet. 2019;138:257-269.

[17] Van-Gils J, Naudion S, Toutain J, et al. Fetal phenotype of Rubinstein-Taybi syndrome caused by CREBBP mutations. Clin Genet. 2019;95:420-426.

\section{Figure Legends}

Figure 1. This piture shows the patient' broad thumbs

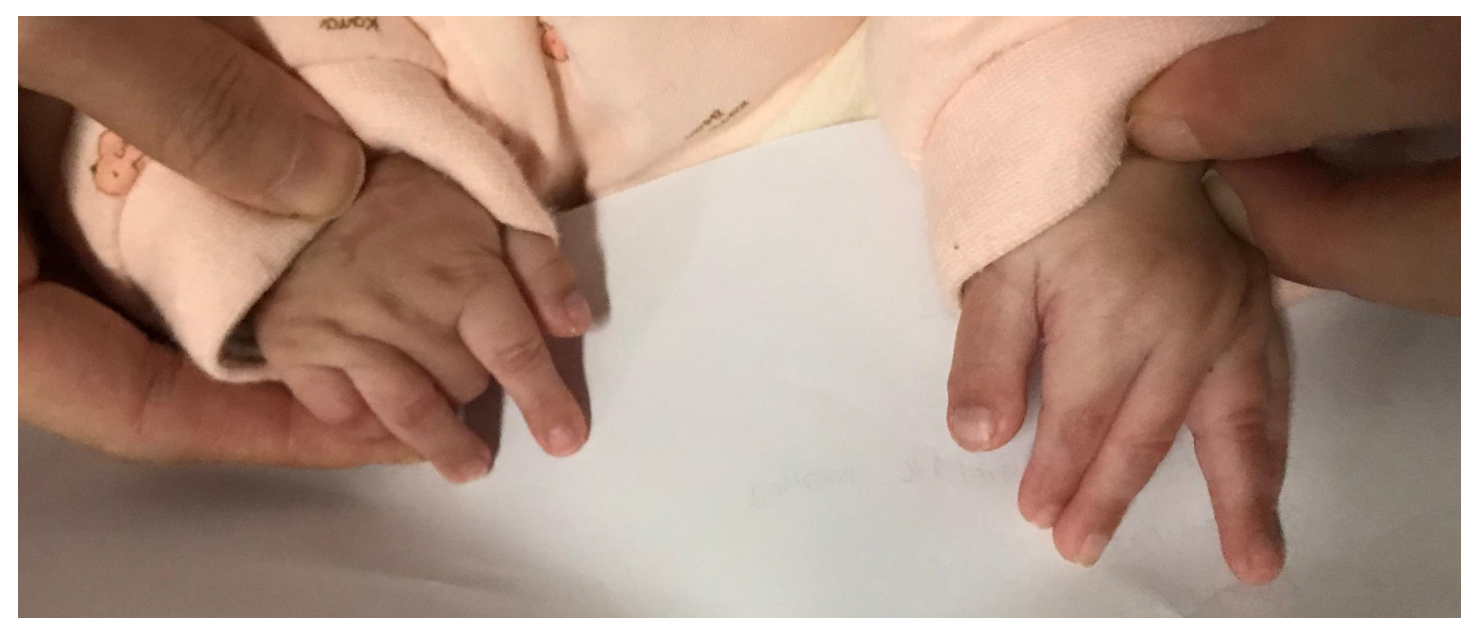

Figure 2. This piture shows the patient' broad toes 


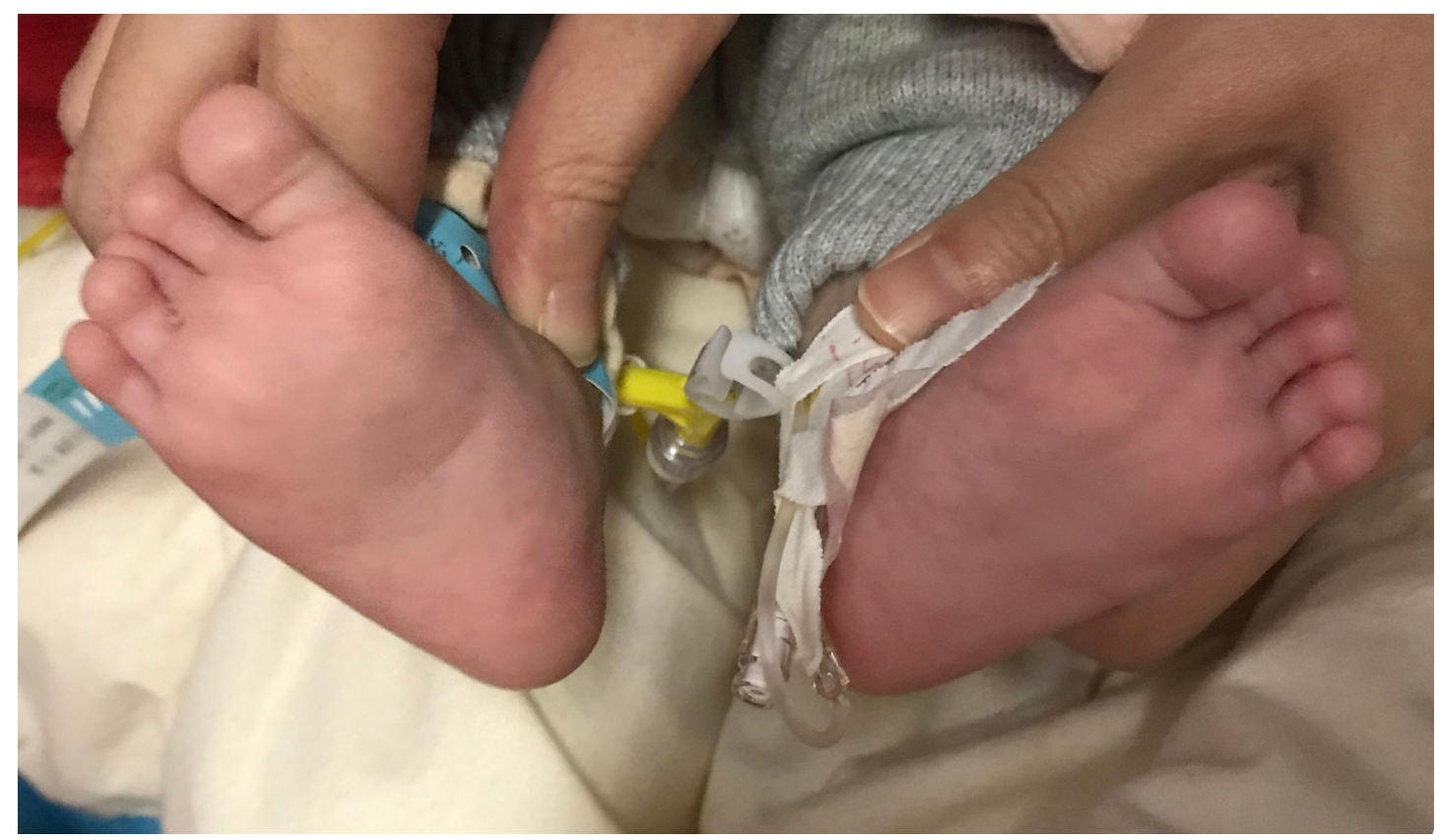

Figure 3. a and bThe patient carries CREBBP gene c.890T> A (p.L297 *) mutation, c and d None

of their parents carried the same mutation

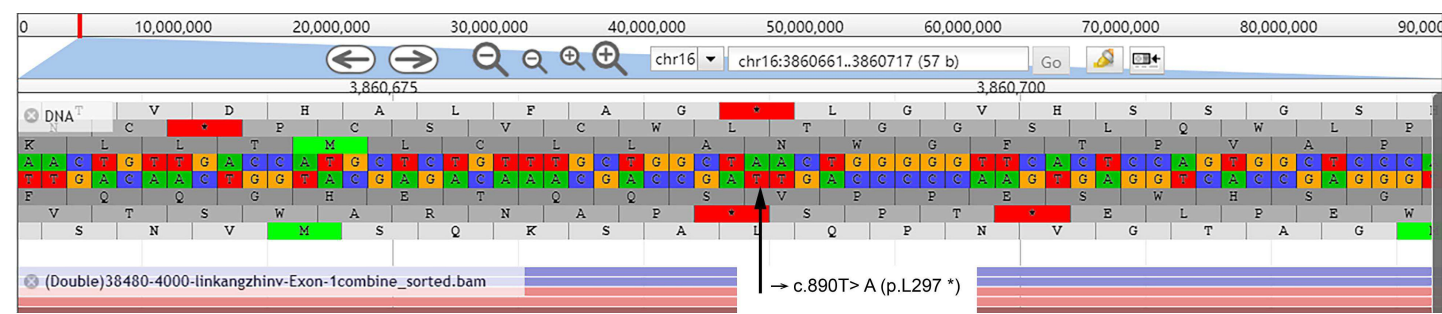

a

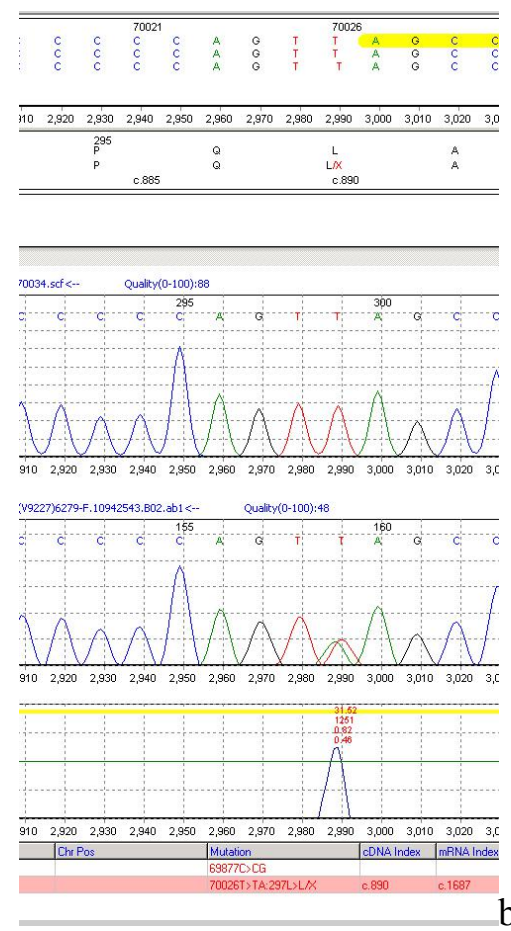




\begin{tabular}{|c|c|c|c|c|c|c|c|c|c|c|}
\hline & 7002 & & 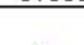 & & & 7002 & & $\cdots$ & & \\
\hline & c & C & A & $G$ & $T$ & $T$ & A & $G$ & C & C \\
\hline & C & C & A & $G$ & T & T & A. & $G$ & C & C \\
\hline & C & c & A & G & $T$ & T & A & G & C & C \\
\hline 30 & 2,990 & 3,000 & 3,010 & 3,020 & 3,030 & 3,040 & 3,050 & 3,060 & 3,070 & $3,0 \varepsilon$ \\
\hline 5 & & & $Q$ & & & L & & & A & \\
\hline & & & $Q$ & & & L & & & A & \\
\hline & c. 885 & & & & & c. 890 & & & & \\
\hline
\end{tabular}

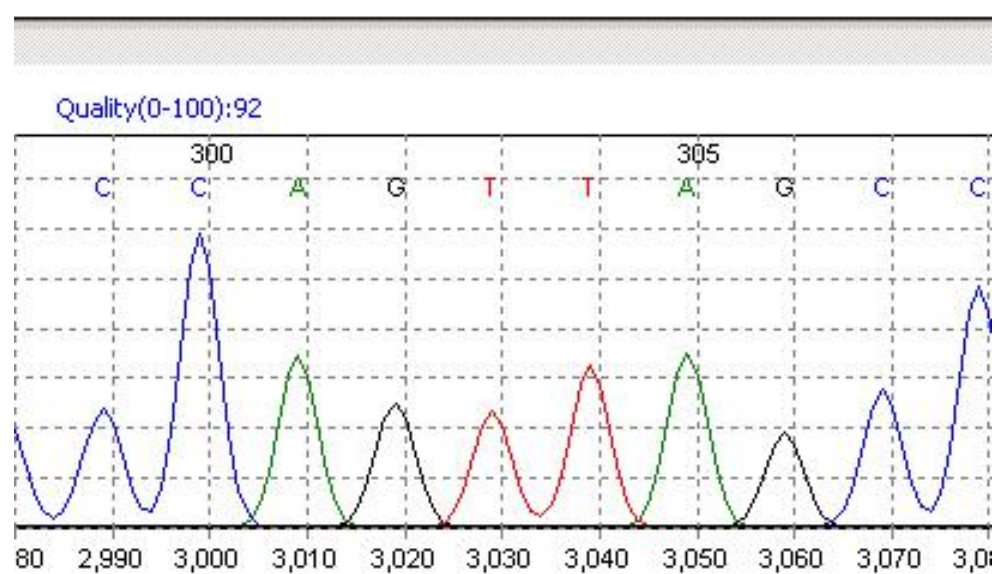

:545.E03.ab1<-- Quality $(0-100): 42$
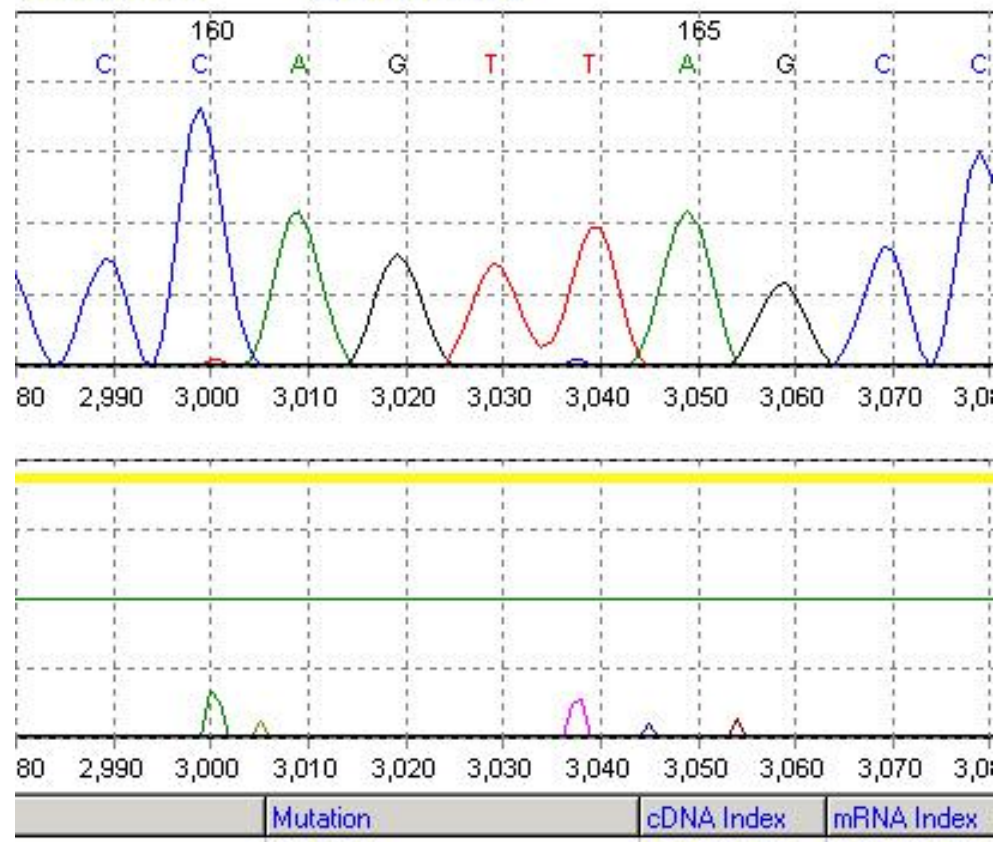

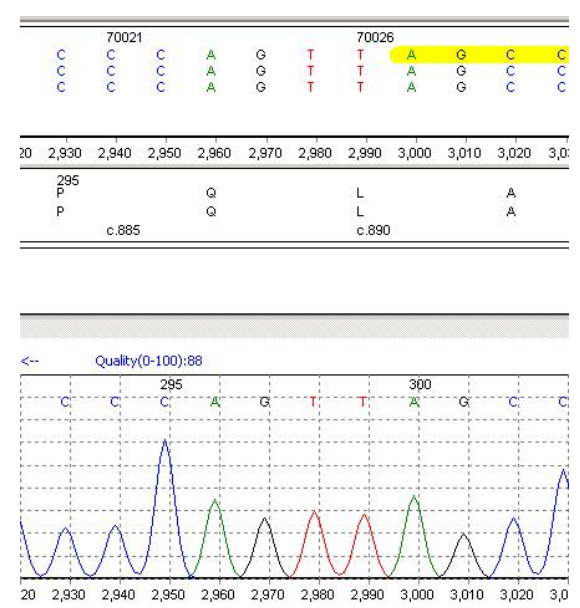

I-F. 10942544.803.ab1<-- Quality(0-100):46
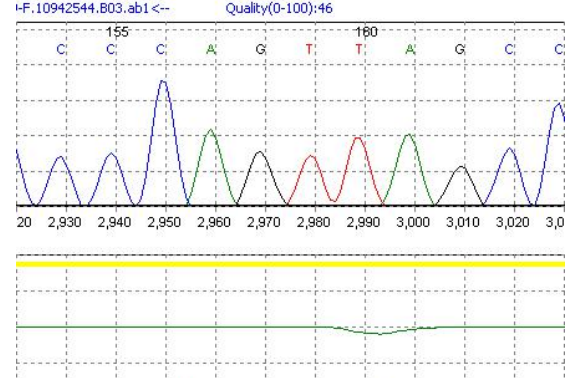

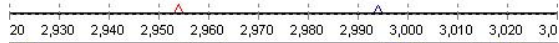
\begin{tabular}{l|l|l|l|l}
\hline hr Pos & Mutation & cDNA Index & miNA Index \\
\hline
\end{tabular}

\begin{tabular}{l|l|l} 
69877C $>C G$ & & \\
$70075 T>T C: 313 D>D / D$ & 0.939 & 0.1736
\end{tabular} d 
Figures

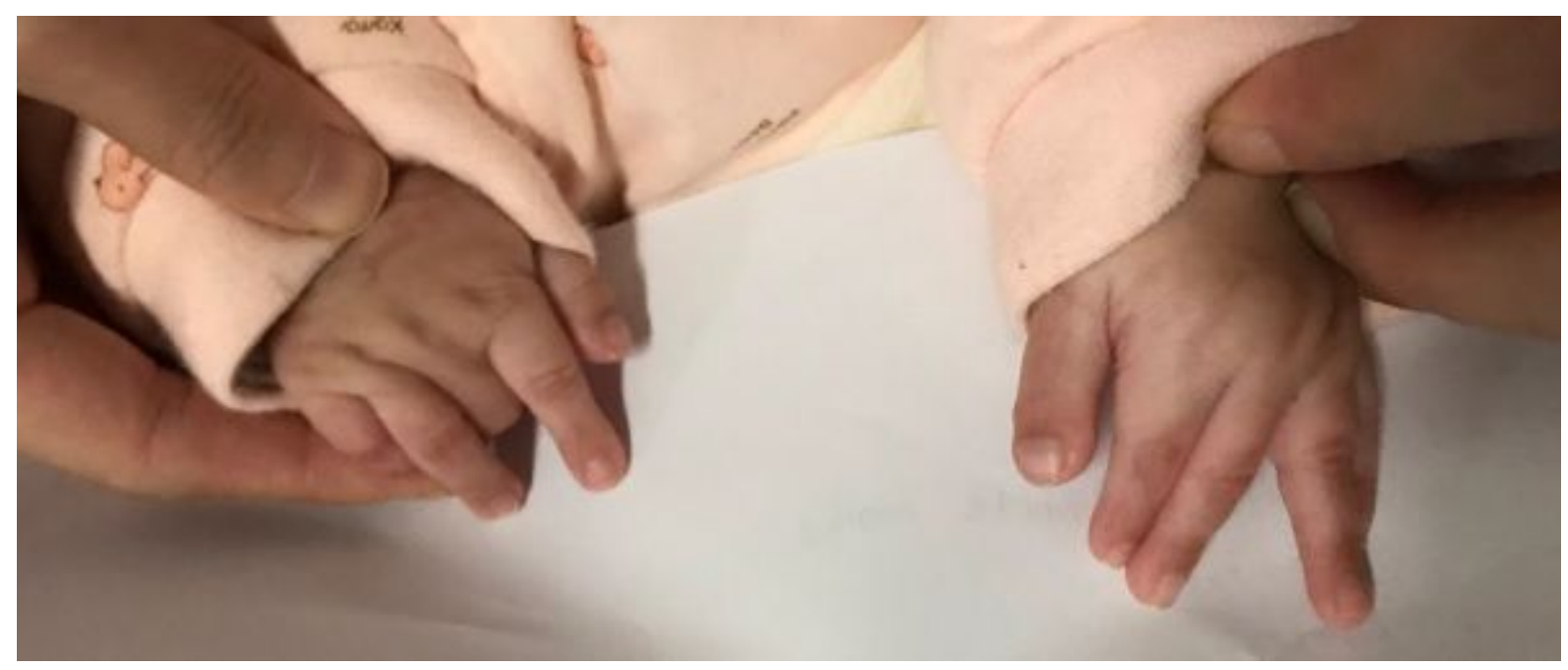

\section{Figure 1}

This picture shows the patient' broad thumbs

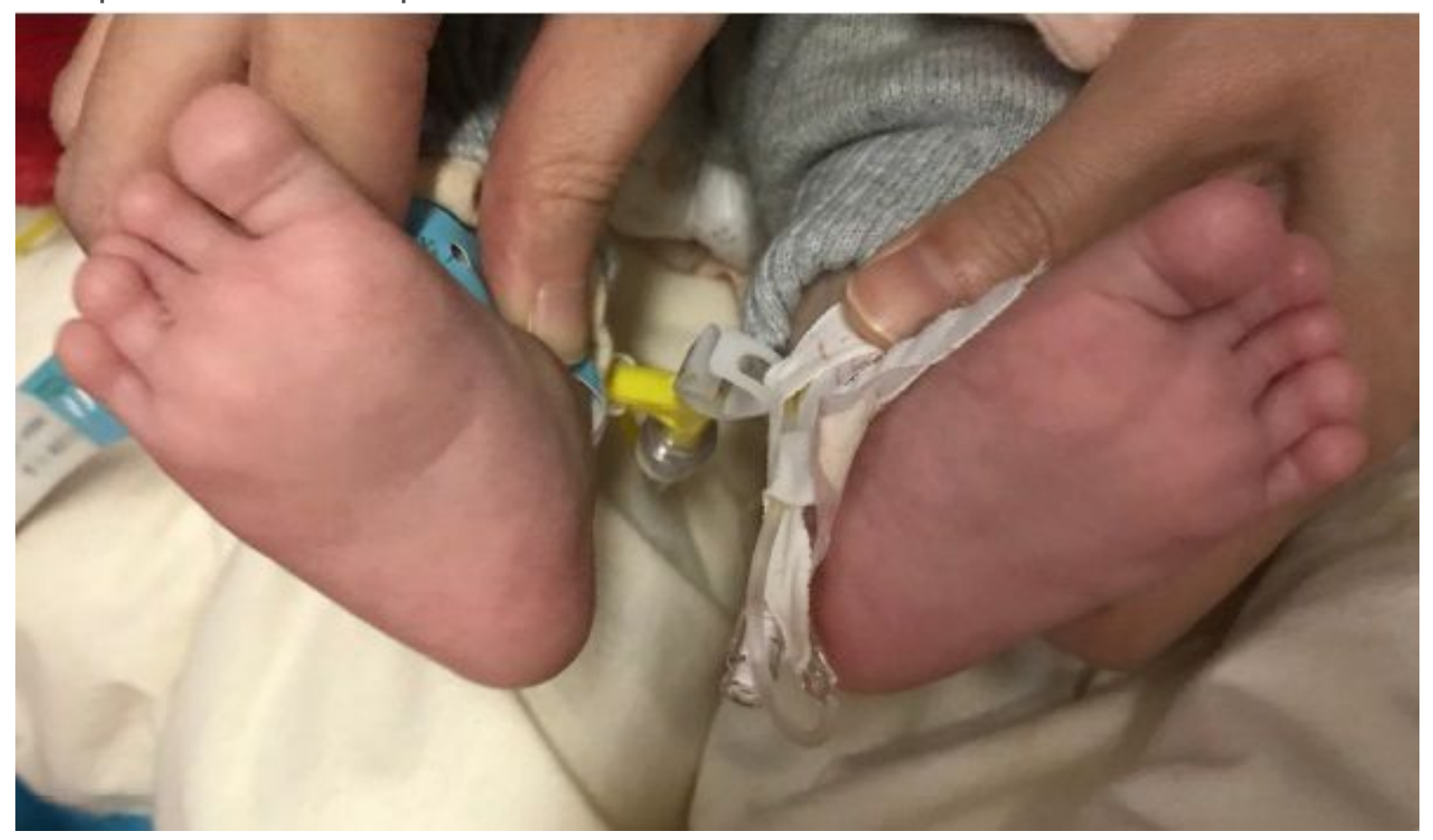

Figure 2

This picture shows the patient' broad toes 


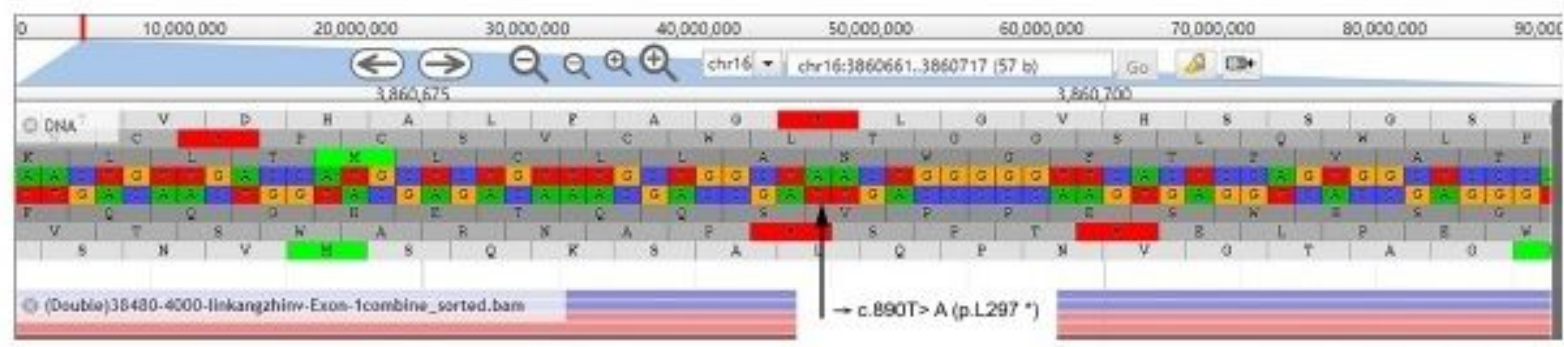

a
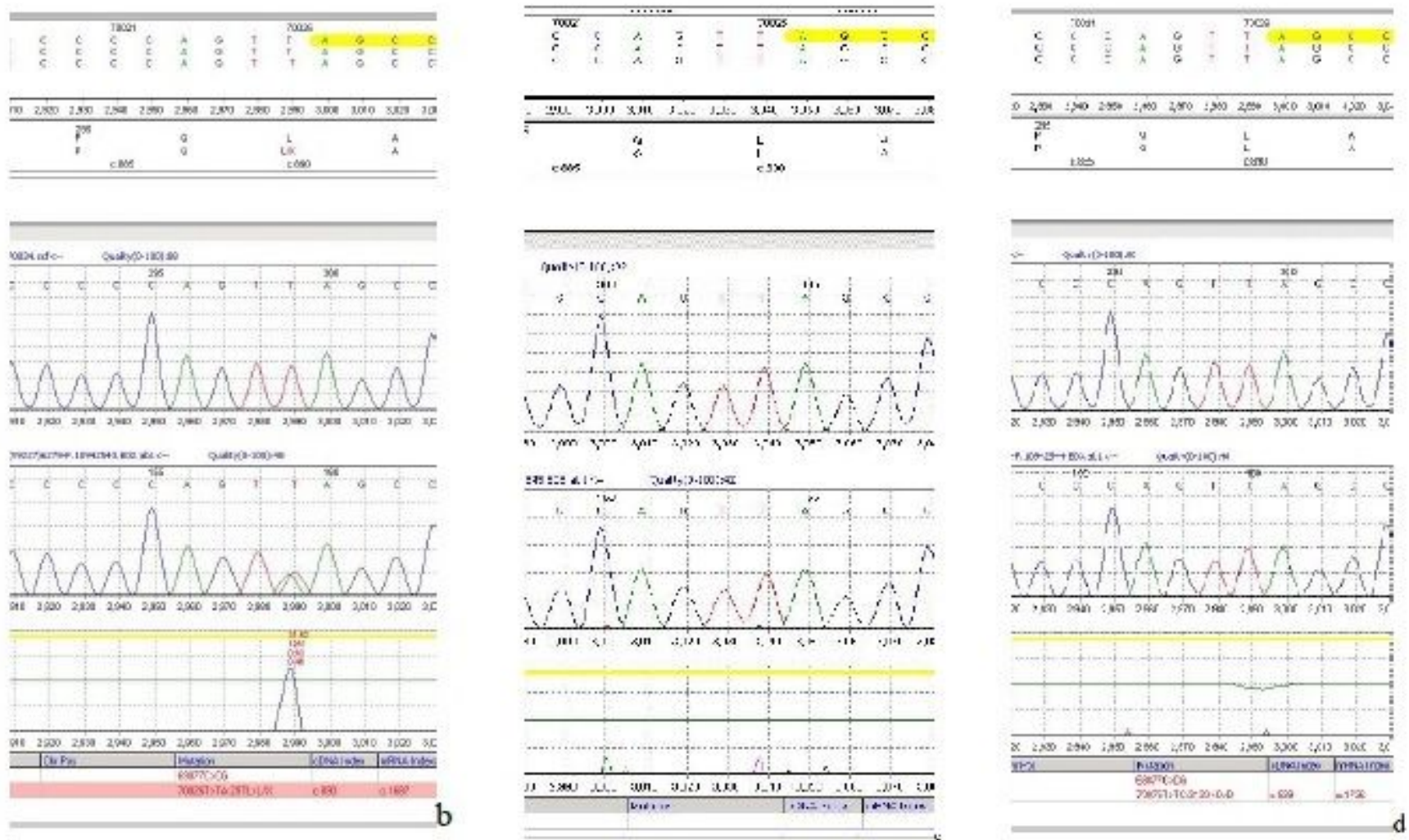

Figure 3

a and bThe patient carries CREBBP gene c.890T > A (p.L297 *) mutation, $c$ and $d$ None of their parents carried the same mutation 